\title{
ACTITUDES HACIA LA DIVULGACIÓN DE LA CIENCIA EN LA INVESTIGACIÓN ACADÉMICA
}

\section{ATTITUDES TOWARD THE DISSEMINATION OF SCIENCE IN ACADEMIC RESEARCH}

\author{
Andrés Castillo Vargas $^{1}$
}

\author{
Fecha de recepción: 26 de julio del 2017 - Fecha de aceptación: 26 de febrero de 2018
}

\begin{abstract}
Resumen
La presente investigación tuvo como objetivo conocer las actitudes del personal docente que investiga en la Universidad de Costa Rica (UCR) en torno a los procesos de comunicación de la ciencia, específicamente la divulgación. Se empleó la Teoría de la Acción Razonada (TAR) con el objetivo de construir un modelo basado en la metodología de ecuaciones estructurales, que permitiera identificar factores que facilitaron la intención de realizar conductas de divulgación en el personal investigador universitario de la UCR. Entre los principales hallazgos, se identifica una valoración positiva en el discurso actitudinal en torno a la divulgación de la ciencia que no implica necesariamente un correlato práctico, así como una predilección por realizar actividades de comunicación oral frente a las de carácter escrito.

Palabras clave: Actitudes, Ciencia, Divulgación, Modelos de ecuaciones estructurales, Teoría de la acción razonada.
\end{abstract}

\begin{abstract}
This research had as main objective to know what were the attitudes around communication processes of science, on the teachers who research at the University of Costa Rica (UCR), specifically the dissemination processes. To do this, we used the Theory of Reasoned Action (TAR) with the aim of building a system based on the methodology of structural equations, which allows identifying the factors that facilitated the intention of performing dissemination around communication processes of science on the researcher personnel working of the UCR. Among the main findings, a positive assessment on the attitudinal discourse on the dissemination and popularization of science is identified. However, this assessment does not necessarily imply a practical correlate. Similarly, a preference for oral communication activities against the written character.
\end{abstract}

Key Words: Attitudes, Science, Dissemination, Structural equation models, Theory of planned behavior.

\section{Introducción}

La creciente importancia de la comunicación en la validación del conocimiento y su valoración como una forma novedosa de promover la cultura científica y la participación ciudadana en temas de ciencia y tecnología (Rivera, 2002), ha revelado la necesidad de hacer comprensible a la sociedad en general los procedimientos y resultados que dentro de las comunidades científicas se realizan.

Actualmente en la esfera académica es preciso no sólo ser buen o buena docente e investigador o investigadora, sino también generar procesos de gestión y transferencia del conocimiento. Es decir, las demandas actuales dentro de la sociedad del conocimiento permiten identificar la necesidad de

1 Instituto de Investigaciones Psicologicas, Universidad de Costa Rica, andres.castillo@ucr.ac.cr 
aumentar las competencias actitudinales y promover un cambio de mentalidad del personal investigador en lo relativo a la comunicación y divulgación de la ciencia.

Considerando este panorama, emergen una serie de interrogantes que vale la pena plantearse: ¿Poseer un claro conocimiento en torno a las ventajas de la divulgación para la investigación académica facilitaría la participación en dicho proceso?, ¿La visibilidad mediática del personal científico-académico contribuye a recaudar fondos para sus investigaciones?, ¿Es la divulgación de la ciencia un proceso de comunicación poco reconocido por la comunidad académica?

Estas y otras preguntas serán analizadas en el presente artículo, para ello se realizó una investigación que buscaba describir las actitudes que el personal investigador de la UCR presentaba en torno a la divulgación científica, con el objetivo de construir un modelo de ecuaciones estructurales que permitiera identificar los factores que facilitaron la intención conductual para realizar actividades de divulgación.

Las actitudes a analizar refieren no sólo a procesos teóricos y prácticos de la comunicación de la ciencia, sino también a procesos intangibles de relativa novedad y reconocimiento en el desarrollo del capital intelectual de la investigación académica. En este sentido, la divulgación se entenderá como la forma de comunicación dirigida a que diferentes esferas de la población posean un acceso fácil, rápido y veraz a información científica de primera mano (Rivera, 2002; Martín \& Rey, 2007).

A pesar de la relevancia que ha adquirido paulatinamente la divulgación, su principal problema asociado ha sido que gran parte de la comunidad científica aún la considera un proceso de poca valía e importancia, restándole con ello legitimidad (Martín \& Rey, 2007; Martínez, 2008). Es por estas razones, que el presente artículo pretende cubrir un vacío de conocimiento relativo a las posibilidades de la comunicación de la ciencia por parte de la comunidad científica y aportar un nuevo marco de comprensión en torno a los procesos de divulgación.

\section{La divulgación de la ciencia: un diálogo con la sociedad}

Comunicar la ciencia es una actividad imprescindible para el desarrollo de la misma. Sin embargo, las particularidades que la caracterizan la convierten en una actividad compleja, plagada de diversos recorridos y escenarios que en muchas ocasiones se restringen al ámbito de las personas expertas académicas con un grado de especialización cada vez mayor. Ello origina una brecha no sólo en su relación e intercambio con la sociedad, sino también para con otros y otras especialistas de distintas disciplinas.

A este panorama, se suma la preocupación creciente plasmada en distintas investigaciones (Martín \& Rey, 2007; Martínez, 2008), las cuales sugieren que, si la ciencia no logra ser comunicada adecuadamente y llegar a la sociedad, lo hará la pseudo ciencia, originando una serie de nuevas controversias y dificultades al dar cabida a mitos e ideas falsas como si fueran conocimiento científico válido.

Justo por estos argumentos, se considera relevante analizar las actitudes que posee el personal académico hacia los procesos de comunicación de la ciencia, comprendiendo a este personal como un agente cultural cuya actividad laboral, es en términos generales, valorada en función de su impacto y sus contribuciones (Martín \& Rey, 2007); de modo que conocer sus actitudes frente a la divulgación científica contribuirá a identificar el conocimiento y la estimación que poseen de dicho proceso, además de favorecer el reconocimiento del rol desempeñado por ellos y ellas en el establecimiento de puntos de encuentro comunicativos entre el mundo de la ciencia y la sociedad.

Si bien la divulgación de la ciencia puede realizarse de diversas formas, por razones metodológicas en el presente estudio se brindó especial énfasis a las actividades de divulgación relacionadas con los medios de comunicación tradicionales (prensa escrita, radio y televisión), en las cuales el personal investigador pueda desempeñarse como divulgador de su propio trabajo. 


\section{Las actitudes como reacciones evaluativas}

El concepto actitud ha sido un término difícil de acuñar en la literatura científica debido a la complejidad de su significado y a la discordia que eventualmente se presenta en torno a los componentes que le conforman.

A la luz de los aportes de corrientes cognitivo-conductuales, las actitudes fueron definidas como tendencias evaluativas que una persona o un grupo cultural emitía respecto al grado de aprobación o desaprobación de determinada conducta u objeto (Ajzen, 2002). De modo que comprender a las actitudes como predisposiciones evaluativas influenciadas por la interiorización de normas culturales, lleva a reconocer el carácter tripartito que le diferencia de otros procesos psicosociales. Justamente, Méndez, Valdez, Velázquez, Rivera y Salmerón (2003) concuerdan en que la estructura de las actitudes se distingue por tres componentes principales, a saber:

un componente "cognitivo, [el cual] se refiere a las ideas o información que tiene un individuo respecto del objeto de actitud; [un componente] afectivo, [que] se relaciona con el sentimiento de simpatía o antipatía, respecto del objeto de actitud, y [finalmente, un componente] conductual, [que] indica el comportamiento del individuo respecto al objeto de actitud (p.475).

Los postulados de Méndez et al (2003) coinciden con la posición de Ajzen y Fishbein (1975), quienes consideran que las actitudes son influenciadas por los estados de ánimo y las emociones, al igual que están reguladas por las cogniciones y la volición. Para estos investigadores sociales "el afecto refiere a los sentimientos de una persona hacia algún objeto, persona, tema o evento y a su evaluación de éstos; la cognición denota sus conocimientos, opiniones, creencias y pensamientos respecto al objeto y la volición refiere a sus intenciones de comportamiento y a sus acciones en relación con el objeto o en presencia de éste" (p.12), estas tres variables se encuentran en continúa interrelación conformando el fundamento primordial de las actitudes.

\section{La predicción de intenciones comportamentales mediante la TAR}

La teoría de la acción razonada (Ajzen \& Fishbein, 1975) se centra en la racionalidad del comportamiento, basándose "en el paradigma general de que los seres humanos, antes de actuar, hacen uso sistemático, detallado y racional de la información que poseen sobre las posibles implicaciones de ejecutar una conducta" (Mújica, Guido \& Mercado, 2011, p.78).

Vale la pena señalar que en sus inicios la TAR carecía del componente de control conductual percibido o autoeficacia, el cual fue incorporado años después por el autor como una manera de explicar las críticas que le limitaban solamente a comportamientos volitivos (Mújica et al., 2011), obviando los obstáculos personales y situacionales a los cuales el individuo se enfrentaba al tener la intención de realizar una conducta. Al incluir esta nueva variable, Ajzen (2001, 2002a, 2002b, 2011, 2012) amplió el bagaje conceptual de la teoría renombrándola como teoría de la acción o conducta planificada, incorporando así el estudio de comportamientos que puedan estar o no, bajo el control de la persona. No obstante, en la práctica un número significativo de investigadores e investigadoras ha seguido utilizando su nombre original, pues realmente no existe una resignificación de la teoría en sí misma, sino más bien la inclusión de una nueva variable que le brinda mayor solidez predictiva (Ajzen, Joyce, Sheikh \& Gilbert, 2011).

De acuerdo con esta teoría, la cual se emplea en este estudio; la acción humana está influenciada por tres grandes factores: la evaluación positiva o negativa de la conducta (actitud hacia la conducta), la percepción de la presión social para realizar o no la conducta (norma subjetiva) y la capacidad percibida para realizar la conducta (autoeficacia o control conductual percibido). La combinación de estos tres factores da como resultado la formación de la intención conductual, la cual es considerada el antecedente inmediato en la ejecución de una conducta (Ajzen \& Gilbert, 2008). Esta teoría postula que las actitudes, 
normas subjetivas y control conductual percibido son los principales determinantes de las intenciones de comportamiento y por ende de la conducta real.

Sobre esta misma línea discursiva, Reyes (2007) es enfático en resaltar que el objetivo de la TAR no es la predicción en sí de la conducta, sino más bien la predicción probabilística y comprensión de los determinantes que hacen posible dicha conducta; aspecto de especial interés para este estudio, dada la intención de vincular la TAR con la metodología de modelos de ecuaciones estructurales.

\section{Método}

La presente investigación se concibió como un estudio cuantitativo transversal de una sola medición (Anguera, Arnau, Ato, Martínez, Pascual \& Vallejo, 1998; Barrantes, 2007). En términos de su alcance, constituye una investigación tanto descriptiva como correlacional; en relación con las variables de análisis, estas están constituidas por los componentes que subyacen y caracterizan a las actitudes y a la conducta de acuerdo al modelo de expectativa-valor y a la TAR desarrollada por Ajzen y Fishbein (1975). Específicamente, las variables para este estudio son: las creencias o normas subjetivas, las valoraciones actitudinales propiamente dichas, el control conductual percibido y la intención -en calidad de antecedente inmediato a la ejecución de un comportamiento-; las cuales fungen como variables latentes (Kerliger \& Lee, 2002). De igual modo, en calidad de variables observables que delimitan el campo de estudio, se contemplan las conductas vinculadas con los procesos de divulgación de la ciencia.

Para el caso específico de este estudio, las actitudes fueron concebidas como aquellas reacciones subjetivas que se disponen a responder con algún nivel de agrado o desagrado hacia un objeto en particular (Ajzen \& Gilbert, 2008), las cuales pueden ser influenciadas por los estados de ánimo y las emociones. Las normas subjetivas son aquellas creencias, factores cognitivos y/o motivacionales influenciados por el contexto - presión social percibida-, que conforman la expectativa o probabilidad subjetiva que un referente grupal o individual apruebe o desapruebe el llevar a cabo la conducta que se está evaluando (Ajzen \& Gilbert, 2008).

El control conductual se asumió como el sentido de autoeficacia percibida de acuerdo con la valoración de dos tipos de obstáculos, los personales: falta de habilidades o competencias para realizar la conducta y los situacionales: oportunidades y recursos disponibles en el contexto para ejecutar la conducta. Por otra parte, la intención es definida como el antecedente inmediato para la ejecución e intensidad de una acción determinada, es decir, es la proclividad (grado o probabilidad en que una persona pueda realizar una conducta) de efectuar un comportamiento específico de acuerdo con las creencias, evaluaciones y control percibido que se posean en torno a este (Ajzen \& Gilbert, 2008).

Finalmente, las conductas vinculadas con los procesos de comunicación científica fueron concebidas teóricamente como "las maneras en las cuales se demuestran los conocimientos y las actitudes a través de acciones" (Kaliyaperumal, 2004, p.7), esto es, comportamientos habituales o rutinarios que el personal investigador realiza para dar a conocer el resultado, los procedimientos y/o implicaciones de sus investigaciones a la sociedad en general.

Dada la variabilidad de actividades de divulgación de la ciencia que existen en la actualidad y de acuerdo con investigaciones previas (Martín \& Rey, 2007), el presente estudio les delimitó -con fines operacionales y predictivos- a un número específico de conductas. A saber, artículos de prensa en periódicos o revistas no científicas, expo-ferias, entrevistas en calidad de personas expertas, libros de divulgación científica, realización de cursos de extensión docente, conferencias de prensa, participación en museos científicos, programas de radio, programas de televisión, divulgación electrónica en blogs, redes sociales u otro tipo de sitios web institucionales o públicos, jornadas de puertas abiertas y conferencias públicas. 


\section{Hipótesis}

$H_{1}$ Existe una asociación positiva entre las creencias normativas hacia la divulgación y las actitudes en torno a la divulgación.

$H_{2}$ Existe una asociación positiva entre las normas subjetivas sobre los procesos de divulgación y el control percibido en torno a la divulgación científica.

$H_{3}$ Conforme aumentan las creencias normativas positivas en torno a la divulgación aumenta la intención de participar en actividades de divulgación.

$\mathrm{H}_{4}$ Existe una asociación positiva entre las actitudes favorables frente a la de divulgación y el control percibido hacia la divulgación.

$H_{5}$ Conforme aumentan las actitudes favorables en torno a la divulgación aumenta la intención a divulgar.

$H_{6} \mathrm{Al}$ aumentar el control percibido hacia la divulgación aumenta la intención de participar en actividades de divulgación de la ciencia.

$H_{7} \mathrm{Al}$ aumentar la intención de participar en actividades de divulgación de la ciencia aumenta la frecuencia de conductas de divulgación.

Considerando estas hipótesis, el modelo de medida establecido se puede observar en la figura 1 , comprendiendo de antemano que $\xi_{1}=$ Creencias entorno a la divulgación $-\xi_{2}=$ Valoraciones personales ante la divulgación- $\xi_{\mathbf{3}}=$ Control Conductual percibido- $\boldsymbol{\eta}_{\mathbf{1}}=$ Intencionalidad Conductual $-\boldsymbol{\eta}_{\mathbf{2}}=$ Conductas de divulgación realizadas.

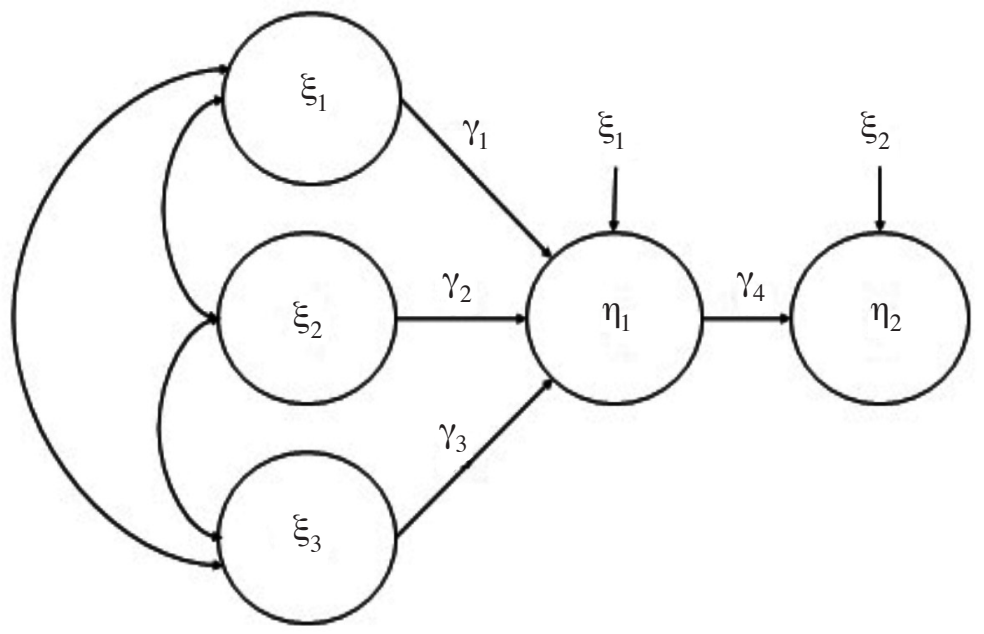

Respecto a la población del estudio, se extrajo una muestra no probabilística por conveniencia de 25 unidades académicas pertenecientes a la UCR (11 correspondientes al área de Ciencias Sociales y 14 correspondientes al área de Ciencias Básicas), en ellas se lograron entrevistar a 171 personas que realizaban labores de investigación científica, 85 mujeres y 86 hombres. Se construyó un cuestionario que contempló tanto preguntas cerradas como preguntas abiertas, así como reactivos de pregunta en escalas tipo Likert y de diferencial semántico; dicho cuestionario estuvo conformado por 6 escalas: escala de actitudes hacia la divulgación, escala de dificultades para divulgar, escala de norma subjetiva para la 
divulgación, escala de control percibido en torno a la divulgación, escala de intenciones para divulgar, escala de diferencial semántico hacia la divulgación, el mismo fue enviado de forma online por medio de la plataforma Limesurvey.

El cuestionario incluyó una fórmula de consentimiento informado, en la cual se especificaban los objetivos, riesgos y alcances de la eventual participación en la investigación; dicha fórmula es una vía para asegurar la protección de las personas participantes ante cualquier eventualidad que pudiera presentarse. Asimismo, se aseguró la confidencialidad, el anonimato y la libertad de negarse a participar en la administración del cuestionario o a suspender su eventual participación.

Para certificar la fiabilidad se realizaron una serie de análisis estadísticos con el objetivo de asegurar la consistencia interna del instrumento, entre dichos análisis sobresale el empleo del Alfa de Cronbach. Los índices Alfa reportados aseguraron la adecuada confiabilidad de cada una de las escalas utilizadas, específicamente en la escala de actitudes (15 ítems) $\alpha$ : .896, en la de dificultades (6 ítems) $\alpha: .634$, norma subjetiva (11 ítems) $\alpha: .873$, control percibido (7 ítems) $\alpha: .865$, intenciones (11 ítems) $\alpha$ : .898 y diferencial semántico (5 ítems) $\alpha: .857$.

Del mismo modo, de acuerdo con la naturaleza de los datos, se procedió a la realización de distintos análisis, entre ellos sobresalen estadísticas descriptivas: distribución de frecuencias, análisis de contingencias, revisión de medidas de tendencia central y de variabilidad, además de análisis de correlación, para finalizar con el reporte de dicha información bajo un formato de elaboración de tablas y gráficas.

También se realizó un proceso cualitativo de validación de expertos (as), en el cual 11 especialistas analizaron la estructura del instrumento con la finalidad de depurar el cuestionario de acuerdo con su experticia. Para certificar la validez de constructo se realizó un análisis de factores por medio del paquete estadístico SPSS, que contribuyó a identificar la correlación existente entre los ítems, así como la correspondencia teórica entre las variables a investigar. Finalmente, se realizó una prueba piloto del cuestionario, dirigida a personal investigador de la UCR que poseía características similares a la población meta.

\section{Descripción de resultados}

La muestra final estuvo conformada por 85 mujeres y 86 hombres, el $49.7 \%$ y $50.3 \%$ de la muestra respectivamente, la media de edad fue de $\mathrm{M}=42.98$ años, $\mathrm{DE}=10.74$, con un rango entre 25 años y 70 años. Sobre el tiempo de laborar en la Universidad, se obtuvo una media de $\mathrm{M}=9.89$ años, DE = 9.54, con un rango entre 1 año y 41 años. En torno al grado académico de cada participante, se destaca que $42.7 \%$ (73 participantes) posee un doctorado académico, seguido de un 33.9\% (58 participantes) de la muestra con una titulación de maestría.

En relación con el desarrollo de iniciativas que promuevan la comunicación de la ciencia, se expone que el 20.5\% (35 participantes) de la muestra reporta que en su Centro o Instituto de investigación se cuenta con un programa o proyecto específico encargado de desarrollar y promover procesos de comunicación de la ciencia, el 31\% (53 participantes) reporta no saber si existe dicho programa, mientras que el $48.5 \%$-el porcentaje mayor- (83 participantes) reporta que no existe ninguna iniciativa de este tipo en su unidad académica. Aunado a ello, el 95,3\% del personal académico encuestado (163 participantes) también reportó nunca haber participado en alguna actividad de capacitación y/o educación sobre redacción de artículos de prensa u otras actividades dirigidas a públicos no especialistas.

\section{Actitudes, norma subjetiva y control conductual percibido hacia la divulgación de la ciencia}

Las actitudes del personal investigador hacia la divulgación de la ciencia, fueron indagadas a partir de una escala de 15 preguntas cerradas y una escala de diferencial semántico conformada por 5 reactivos. En términos generales, se aprecia que la mayor parte del personal investigador evaluado posee una actitud positiva hacia la divulgación, resaltando los beneficios que la misma tiene en el desarrollo 
de la investigación y en el fomento de la participación ciudadana en los temas científicos; sobresalen los ítems "DV21. Contribuye al progreso social" (63.2\%), "DV20. El personal investigador tiene que conocer las diferentes audiencias a las cuales puede dirigirse con el fin de divulgar sus resultados" (60.2\%), "DV17. La divulgación es una actividad intrínseca a la labor investigadora" (58.5\%), "DV25. Promueve el conocimiento basado en las evidencias" (57.9\%) y el "DV14. Debo hacer llegar los resultados de mis investigaciones a diferentes públicos" (57.3\%); los cuales poseen los niveles más altos de consenso (totalmente de acuerdo).

Como dato curioso, se puede mencionar el nivel de consenso del ítem "DV17. La divulgación es una actividad intrínseca a la labor investigadora" (58.5\% estuvo totalmente de acuerdo); el cual es bastante alto, no obstante, si comparamos esta valoración actitudinal con otras interrogantes del cuestionario, notaremos que contar con una actitud positiva ante la divulgación no asegura que todo el personal investigador posea la capacitación suficiente para realizar dichas actividades o pueda en su defecto dedicarse a este campo. Tal aseveración, puede ser respaldada por la respuesta al ítem "DV19. Todo el personal investigador puede desempeñarse como un buen divulgador de la ciencia", en donde el porcentaje más alto de respuesta, 33.3\%; afirma estar poco de acuerdo con esta afirmación; asimismo en los ítems IG 16 e IG 17 que señalan que el 95.3\% del personal académico no posee capacitación en torno a la redacción de artículos de prensa u otras actividades de comunicación dirigidas a públicos diversos y el $94.2 \%$ carece de capacitación y/o educación que les ayude a relacionarse con profesionales en periodismo, respectivamente.

De primera entrada, las aproximaciones y esquemas evaluativos anteriores parecieran contradictorios, sin embargo, los mismos pueden comprenderse desde el posicionamiento epistemológico desarrollado en la presente investigación, el cual señala que una actitud positiva no indica necesariamente que la persona pueda o desarrolle una conducta determinada, pues también dependerá de su sentido de autoeficacia, de la norma subjetiva y del impacto de las mismas en su intención conductual. Por lo cual, la reflexión en torno a dichos esquemas debe dirigirse más bien al análisis de los factores que facilitan u obstaculizan la divulgación dentro de una comunidad académica determinada, como lo es en este caso la UCR.

En el diferencial semántico realizado se presenta un número significativo de respuestas con un alto nivel de consenso positivo, específicamente 4 de los 5 ítems que conformaron la escala reportan más de un $70 \%$ de acuerdo (totalmente de acuerdo). A la luz de esta serie de respuestas, la divulgación es valorada como importante $(91.8 \%)$, valiosa $(91.2 \%)$, productiva $(82.8 \%)$ y satisfactoria $(73.4 \%)$. Además, es calificada por un porcentaje importante de personas como un proceso accesible (64.9\%).

Con relación a las normas subjetivas del personal investigador hacia la divulgación de la ciencia, estas fueron evaluadas a partir de una escala de 11 preguntas cerradas. En términos generales, ellos y ellas manifiestan percibir que su Centro o Instituto de Investigación o la Universidad en sí, promueven desde lo formal la relevancia de la divulgación; es decir, desde el discurso oficial se recomienda que el personal académico se involucre en actividades de divulgación científica; algunas respuestas que sustentan esta afirmación, las encontramos en los siguientes ítems, las cuales se ubicaron en la categoría muy de acuerdo, "DV40. Mis pares científicos consideran que la divulgación trae consigo el aumento de las relaciones con otros profesionales" (39.2\%), "DV39. La normativa universitaria estipula que los procesos de divulgación son fundamentales para el desarrollo de la investigación científica" (38.0\%), "DV35. El Centro o Instituto al que pertenezco promueve la divulgación de los resultados de investigación a públicos diversos" (35.7\%).

No obstante, a pesar de esta postura oficial, el personal académico encuestado señala también el poco apoyo que percibe para que dicho discurso se traduzca en beneficios reales en su carrera académica, por lo tanto, podría decirse que la influencia que ejercen estos referentes es más de índole normativa que práctica. Algunos ítems que ejemplifican esta disyuntiva y que se ubican en la categoría poco de acuerdo, son el "DV41. La Universidad me ha brindado la suficiente motivación para divulgar mis investigaciones" (43.3\%), "DV36. Cuento con incentivos institucionales para participar en actividades de divulgación científica" (42.1\%) y el "DV38. Mi Centro o Instituto considera que la divulgación es 
una forma de captar recursos para la investigación" (36.8\%); puede afirmarse que la respuesta a estos ítems denota la falta de reconocimiento de la divulgación como un intangible de gran valor en el capital intelectual de una Universidad pública, como lo es la UCR.

Además, sobresale también el ítem "DV37. El participar en actividades de divulgación me ha sido de ayuda para ascender en régimen académico" (39.8\%), que es el único ítem de dicha escala que su ubica en la categoría nada de acuerdo, lo cual deja entrever como para el personal investigador evaluado, el sistema de meritazgo utilizado por la UCR (régimen académico) no premia o puntúa positivamente la realización de actividades de divulgación. Este dato, conlleva a reflexionar en torno a la necesidad de modificar los sistemas actuales de valoración universitarios, con el fin de incluir y visualizar la importancia del proceso de divulgación de la ciencia en el crecimiento académico y en el fortalecimiento de novedosas líneas de investigación.

El sentido de auto eficacia o control conductual percibido para llevar a cabo conductas de divulgación, fue evaluado con una escala de 7 ítems. Las capacidades que presentan mayor consenso (totalmente de acuerdo) y que el personal científico percibe subjetivamente como propias, se evidencian en los ítems "DV61. Responder preguntas de forma simple" (51.5\%) y el "DV60. Exponer verbalmente mis investigaciones a un público con pocos conocimientos científicos" (47.4\%).

Resulta interesante mencionar la variedad de posiciones en torno al ítem "DV65. Crear un blog de divulgación científica", pues a pesar de contar con un $26.3 \%$ de respuestas que indican estar muy de acuerdo con dicha afirmación, existe un $25.1 \%$ que argumenta estar poco de acuerdo, junto a otro $25.1 \%$ que argumenta estar nada de acuerdo. Este dato, conlleva a especular que un importante número del personal académico encuestado puede no manejar de manera adecuada las nuevas herramientas de divulgación y los recursos electrónicos que la tecnología actual pone a disposición.

Por otro lado, la intención para llevar a cabo conductas de divulgación fue evaluada con una escala de 11 ítems. El dato que más llama la atención son los ítems "DV69 Divulgar mis investigaciones en programas de televisión de acceso popular" (39.2\%), "DV73 Redactar libros de divulgación" (29.8\%), "DV74 Divulgar los resultados de mis investigaciones por medio de la ejecución de cursos de extensión docente" (27.5\%), "DV75 Participar en las expo-ferias organizadas por la Universidad" (30.4\%),"DV71 Crear un sitio web con el fin de divulgar información sobre mi investigación" (35.7\%) y "DV76 Divulgar mis investigaciones en programas populares de radio" (32.7\%), los cuales se ubican mayoritariamente (\%) en la categoría poco de acuerdo.

Las respuestas a dichos ítems, reflejan que el personal investigador posee pocas intenciones de divulgar en medios de comunicación como la televisión, la radio y sitios web, así como redactar libros de divulgación o participar en expo ferias. Este hallazgo, podría sugerir cierta desconfianza del personal académico en los grandes medios de comunicación, o en su defecto, podría indicar que desean divulgar sus investigaciones, pero no saben realmente cómo hacerlo y con qué recursos cuentan.

Otra posible hipótesis que podría justificar este dato, es quizás la poca familiaridad que parece tener el personal investigador con medios electrónicos destinados a la divulgación y su escaso contacto con profesionales dedicados a la comunicación, situación que puede generarles cierta apatía.

\section{Modelo de ecuaciones estructurales hacia la divulgación de la ciencia}

A continuación, se describe por medio de un modelo de ecuaciones estructurales, la asociación existente entre las variables actitudes, norma subjetiva y control percibido con la intención de realizar conductas vinculadas a la divulgación de la ciencia. Para la estimación del modelo hacia la divulgación se incluyeron todos los ítems correspondientes para cada constructo, no obstante, se agruparon en parcelas para aumentar el ajuste del modelo ya que lo importante a la luz de las hipótesis planteadas eran las relaciones estructurales entre los constructos.

La técnica de parcelas consiste en añadir juntos un número determinado de ítems, sumados o promediados con el fin de obtener un indicador que representará a varios ítems. Esta estrategia es de 
cuantiosa utilidad según Little, Cunningham, Shahar y Widaman (2002) cuando se busca trazar un modelo más parsimonioso y lo que interesa principalmente es la relación entre las variables latentes (Little, Rhemtulla, Gibson \& Schoemann, 2013).

Para la creación de las parcelas incluidas en el modelo, se siguieron las recomendaciones de Little et al. (2002) quienes recomiendan el balance de los ítems por parcela. Como mencionan estos autores, uno de los objetivos es construir parcelas teniendo en cuenta el balance entre dificultad y discriminación (intercepto y pendiente) de los ítems; para ello, se trazó primero un análisis factorial confirmatorio, seguidamente con base en este análisis se establecieron tres parcelas que contienen los tres ítems con mayor carga factorial, la primera parcela contiene el mejor de los ítems según su carga factorial, así que se procedió a incluir seguidamente, el primer ítem con la carga factorial más baja en la primer parcela, posteriormente el segundo ítem con la carga factorial más baja fue incluido en la segunda parcela y el tercer ítem con la carga factorial más baja fue añadido en la tercera parcela. Subsecuentemente, se repite el mismo procedimiento pero en esta ocasión se buscan nuevamente los mejores ítems y se añaden según se hizo inicialmente, hasta obtener parcelas balanceadas según su carga factorial (o su discriminación). Los interceptos de los ítems no fueron tomados en cuenta dado que la dificultad del ítem no es un parámetro relevante en el contexto de la presente investigación.

Las parcelas por constructo estuvieron conformadas de la siguiente manera, Actitud hacia la divulgación: actitud 1 (ítems DV27, DV28, DV24, DV17, DV15), actitud 2 (ítems DV22, DV23, DV20, DV26, DV14) y actitud 3 (ítems DV16, DV21, DV25, DV19, DV18); Norma subjetiva hacia la divulgación: normasub 1 (ítems DV42, DV36, DV39, DV45), normasub 2 (ítems DV40, DV41, DV44, DV37) y normasub 3 (ítems DV35, DV43, DV38); Control conductual percibido hacia la divulgación: control 1 (ítems DV60, DV63, DV65), control 2 (ítems DV61, DV64) y control 3 (ítems DV59, DV62); Intención hacia la divulgación: intención 1 (ítems DV70, DV66, DV75, DV71), intención 2 (ítems DV68, DV69, DV74, DV73) e intención 3 (ítems DV67, DV76, DV72).

Aunado a esto, se utilizaron tres indicadores manifiestos de conducta a saber: participación en programas de radio (ítem DV53), participación en programas de televisión (ítem DV54) y entrevistas ofrecidas a medios periodísticos en calidad de experto (ítem DV56). El modelo demostró una prueba de chi cuadrado de $\chi^{2}{ }_{(83)}=122.91$ el cual fue significativo al nivel del .001; además, se evaluó el ajuste del modelo utilizando el estimado RMSEA el cual fue de .053 con intervalos de confianza al $90 \%$ de .032 hasta .072, resultado que demuestra un ajuste razonable (Browne \& Cudeck ,1992).

Del mismo modo, se realizó una prueba de ajuste perfecto al $90 \%$ de certeza tomando en cuenta el intervalo de confianza inferior, según esta prueba, el modelo estimado no presenta un ajuste perfecto. Seguidamente, se realizó una prueba de ajuste cercano, la cual demostró que se puede sostener la hipótesis de ajuste cercano al nivel de alfa $p<.05$. Asimismo, el indicador TLI fue de .97 y el estimado CFI fue de .97 lo cual permite asumir un buen ajuste (Bentler \& Bonett, 1980; Hu \& Bentler, 1999).

Respecto a las relaciones entre los constructos, se puede apreciar una correlación positiva y significativa de norma subjetiva hacia la divulgación con control percibido hacia la divulgación $(\mathrm{r}=.30$. $p<$ .001) y actitudes hacia la divulgación $(\mathrm{r}=.49, p<.001)$. Asimismo, tal como se esperaba según las hipótesis planteadas, control percibido hacia la divulgación y actitudes hacia la divulgación correlacionaron de manera significativa $(\mathrm{r}=.56, p<.001)$. En esta misma línea, al observar los senderos de regresión se aprecia una predicción significativa de los constructos actitudes hacia la divulgación $(\beta=.25, p<.001)$, control percibido hacia la divulgación $(\beta=.42, p<.001)$ y norma subjetiva hacia la divulgación $(\beta=.25$, $p<.001)$ sobre la intención hacia la divulgación. Asimismo, la intención hacia la divulgación es un predictor significativo de la conducta de divulgación $(\beta=.32, p<.001)$. En total, los constructos exógenos incluidos predicen un 54,3\% de la varianza de intención hacia la divulgación y a su vez, la intención hacia la divulgación explica un 10,3\% de la varianza de conducta de divulgación. De este modo, se concluye que se encontró evidencia favorable para asumir todas las hipótesis planteadas en relación con el modelo estructural de divulgación. 


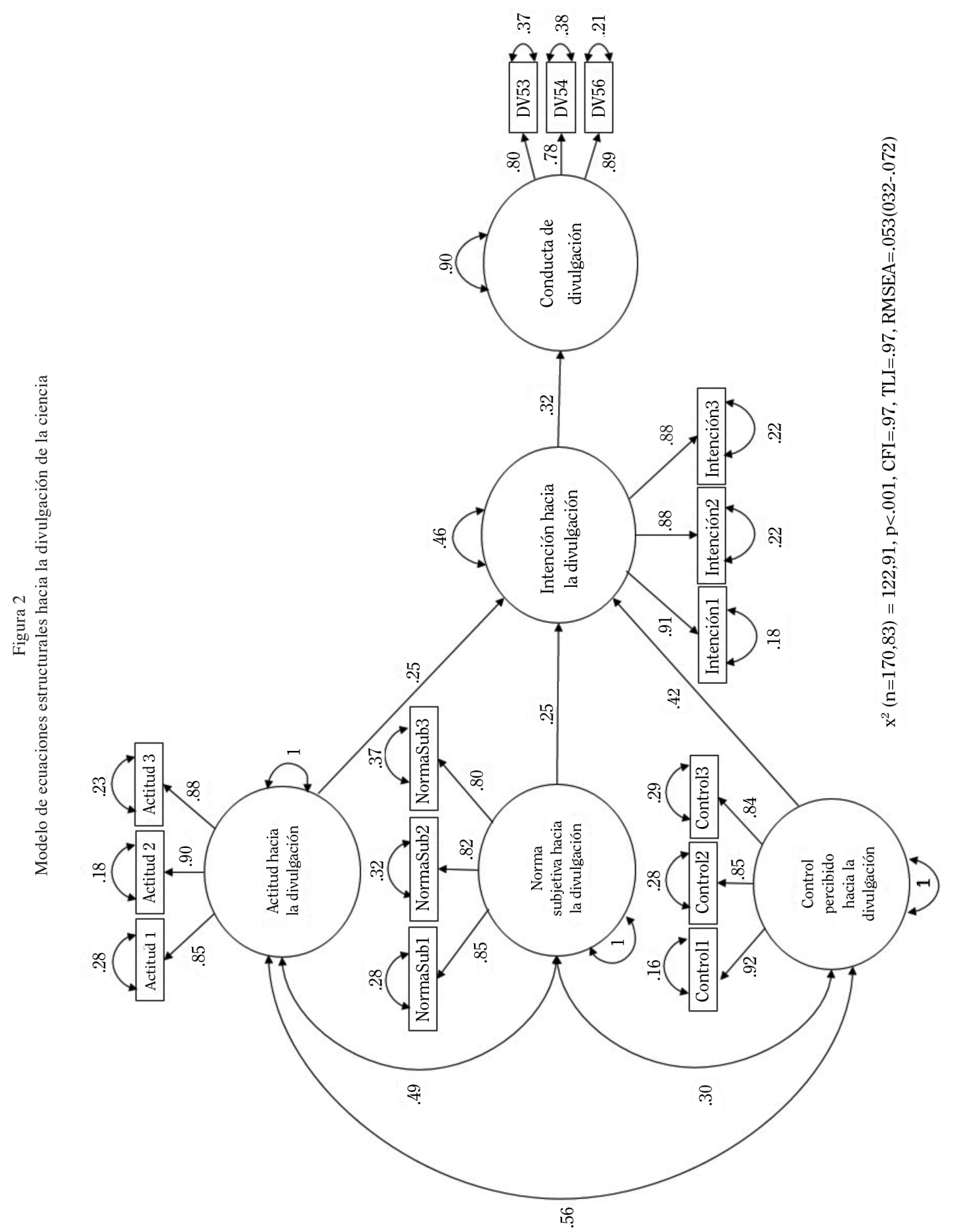


De acuerdo con el modelo de ecuaciones estructurales, figura 2 , se puede afirmar que la actitud $(\beta=.25, p<.001)$, la norma subjetiva $(\beta=.25, p<.001)$ y el control percibido $(\beta=.42, p<.001)$ son facilitadores de la intención a la divulgación, pues todos los constructos mostraron una correlación positiva y significativa (específicamente en relación con la participación en programas de radio, participación en programas de televisión y entrevistas ofrecidas a medios periodísticos en calidad de experto, que fueron las conductas que cargaron en el factor latente del modelo de ecuaciones estructurales realizado). No obstante, el sendero que más sobresale es el de control conductual percibido $(\beta=.42, p<.001)$, por lo cual podría afirmarse que aquellos factores asociados a la autoeficacia o a la percepción subjetiva de tener las capacidades y habilidades personales para participar en actividades de divulgación, juegan un papel especial o de gran relevancia al momento de involucrarse en dichas actividades.

En segundo plano e igualdad de correlación $(\beta=.25, p<.001)$ los factores actitudinales y la presión o el aval de referentes institucionales hacia la divulgación, también son elementos significativos que aumentan la intención por participar en actividades de comunicación científica dirigidas a públicos heterogéneos. Como en el modelo de ecuaciones estructurales estimado no se observaron relaciones negativas, no se pueden mencionar factores que obstaculicen la intención por divulgar la ciencia a partir de dicho modelo, por lo cual, se consultó al personal académico cuáles han sido las principales dificultades que han enfrentado a la hora de querer participar en este tipo de actividades y de acuerdo con lo reportado, los factores principales que dificultan eventualmente la divulgación de la ciencia son la falta de tiempo $(54.8 \%)$ y la falta de reconocimiento por parte de régimen académico (28\%).

Finalmente, las actividades de divulgación que el personal investigador de la UCR reportó realizar con más frecuencia fueron las entrevistas en calidad de expertos a medios periodísticos $(\mathrm{n}=171$, $\mathrm{M}=85, \mathrm{H}=86$ ), las conferencias públicas ( $\mathrm{n}=171, \mathrm{M}=85, \mathrm{H}=86$ ), la divulgación por medio de recursos web ( $n=125, M=57, H=68)$, la publicación de artículos en periódicos ( $n=111, M=41, H=70)$ y la ejecución de cursos de extensión docente ( $\mathrm{n}=118, \mathrm{M}=49, \mathrm{H}=69$ ), evidenciando que son los varones los que reportan mayor cantidad de actividades de divulgación, resaltando además su preferencia por actividades de carácter oral.

\section{Discusión de resultados}

Con respecto a los datos que el personal académico encuestado manifestó en torno a la divulgación de la ciencia, emerge inicialmente una valoración actitudinal muy positiva; la cual evidencia un discurso oficial bastante elaborado que el personal científico ha interiorizado, con la intención de argumentar que la comunicación de la ciencia y en específico la divulgación, forma parte esencial de la investigación académica y de las políticas institucionales. Aunque si se analiza cuidadosamente dicha afirmación, se puede concluir que del discurso oficial a la realidad práctica hay un abismo significativo que sortear, dado el escaso involucramiento del personal académico en actividades de divulgación.

En este sentido, a pesar de calificar a la divulgación como un proceso importante (91.8\%), valioso (91.2\%), productivo (82.8\%), satisfactorio (73.4\%) y accesible (64.9\%); un porcentaje significativo de investigadores e investigadoras afirma que la Universidad les ha brindado poca motivación para divulgar sus investigaciones (43.3\%); además de revelar que cuentan con pocos incentivos institucionales para participar en actividades divulgativas (42.1\%), aunado a que el $95.3 \%$ expresa no poseer capacitación en torno a la redacción de artículos de prensa u otras actividades de comunicación dirigidas a públicos diversos y un 39.8\% afirma estar nada de acuerdo, con que participar en actividades de divulgación le haya sido de ayuda para ascender en régimen académico.

La contradicción expuesta, no sólo evidencia que una valoración positiva de la divulgación en el discurso actitudinal no asegura que existan las condiciones oportunas para su desarrollo; también denota cómo el personal científico carece de procesos de capacitación para tratar de comunicarse con el público no especializado. De la misma forma, el $48.5 \%$ del personal encuestado reportó que en su Centro o Instituto de Investigación no existe ningún programa o proyecto que promueva procesos de 
comunicación científica, lo cual de acuerdo con Loaiza (2012) influye negativamente en el nivel de participación de los científicos en actividades de divulgación.

Considerando este posible efecto negativo vinculado a la carencia de proyectos o programas que promuevan la divulgación de la ciencia, de acuerdo con los resultados encontrados, la presente investigación comparte el criterio de Andrews et al. (2005) y Duarte (2008), quienes suponen que la inclusión del personal científico en actividades de divulgación podría incrementarse si se crean políticas y programas institucionales que impacten sobre los factores que motivan o impiden dicha participación. De igual manera, se considera oportuno que las actividades de comunicación pública de la ciencia fueran catalizadas por una persona coordinadora o grupo central de divulgación, quienes se encargarían de gestionar las oportunidades de comunicación que los Centros e Institutos poseen a nivel institucional y nacional; contribuyendo así al desarrollo de su capital relacional y humano (Edvinson \& Malone, 2000; Bradley, 2003).

Igualmente, el personal científico que emprende actividades de divulgación requiere del respeto de la academia, por el esfuerzo que implica el realizar este tipo de diligencias; dicho respeto solamente se adquirirá si las comunidades de especialistas reevalúan -con carácter de urgencia- sus principios y valores.

Por otra parte, de acuerdo con lo reportado por el personal académico encuestado, los factores principales que dificultaron su participación en actividades de divulgación de la ciencia fueron la falta de tiempo (54.8\%) y la falta de reconocimiento por parte de régimen académico (28\%); factores que se mencionan también en investigaciones precedentes (Corrado, Pooni \& Hartfree, 2000). Al respecto, es notorio nuevamente que dentro de los sistemas de valoración académica la divulgación es una actividad poco reconocida, por lo que quizás el personal científico se dedique a realizar otras funciones que sí les sean puntuadas en su carrera académica y piensen que la divulgación es una actividad "extra" a la cual no pueden dedicarle mucho tiempo.

En este punto es importante resaltar, tal y como se reseñó previamente, que aún gran parte del personal académico que se dedica a la investigación científica, no concibe en el correlato práctico que la divulgación sea una de sus ocupaciones fundamentales, por el contrario, piensan que es una sobrecarga adicional a su trabajo como investigadores e investigadoras. Esta visión es acrecentada, por la carencia de incentivos institucionales, la cual contribuye a que el personal académico catalogue erróneamente como "habilidades blandas" (Gantenberg, 2012), a todas aquellas actividades que no se dirijan a un público especializado.

Esta escisión entre lo actitudinal y lo práctico, fomentada por la misma institucionalidad, justifica en cierto sentido que el personal investigador evaluado conozca los recursos institucionales destinados a la divulgación científica, pero haga un uso limitado de ellos.

Asimismo, la interiorización de una percepción subjetiva negativa en torno a la autoeficacia, es otro elemento a considerar dentro de los obstáculos percibidos por el personal académico en su intención a divulgar, pues se comprobó que existe una correlación negativa entre la intención de realizar actividades de divulgación con la incapacidad para comunicar verbalmente contenidos $(r=-.29, \mathrm{p}<.001)$ y la imposibilidad para reconocer el valor práctico de las investigaciones $(r=-.19, \mathrm{p}<.001)$; lo cual quiere decir, que a mayor percepción de dificultades, es menor la intención de divulgar; aspecto que reafirma la influencia del control conductual percibido (sentido subjetivo de autoeficacia) en la intención de participar en actividades de divulgación científica. Importancia que además fue confirmada por el modelo de ecuaciones estructurales estimado, en el cual el control conductual percibido hacia la divulgación sobresalió como el predictor de mayor correlación en la intención de divulgar $(\beta=.42, \mathrm{p}<.001)$.

Todas estas dificultades percibidas, explicarían a grandes rasgos el por qué la divulgación de la ciencia no es tan "popular" entre las y los académicos como lo es la comunicación dirigida a pares o especialistas (Bentley \& Kyvik, 2011); ofreciendo al mismo tiempo un panorama contextual bastante claro que justificaría el abismo existente entre el discurso oficial académico y la realidad práctica en torno a la divulgación científica. Finalmente, es importante señalar que las entrevistas en calidad de 
expertos-expertas a medios periodísticos y las conferencias públicas fueron las principales actividades de divulgación que el personal académico reportó realizar. Ambos resultados coinciden con los hallazgos de investigaciones anteriores (Torres et al, 2011; Peters, 2008; Agnella et al.; 2012), las cuales evidencian la predilección del personal investigador por actividades orales de divulgación que involucren el contacto cara a cara. Asimismo, este dato deja entrever cómo el personal científico-académico es fuente primaria de información para el personal periodístico, particularidad que le ubica en un rol preferencial en la promoción de la cultura científica.

\section{Conclusiones}

A raíz de un análisis detallado de los resultados se identifica una valoración positiva en el discurso actitudinal en torno a la divulgación de la ciencia, la cual no implica necesariamente un correlato práctico. Del mismo modo. se percibe una desvalorización de las actividades de divulgación dentro de régimen académico, las cuales carecen de popularidad dentro de la comunidad académica.

El personal científico encuestado prefiere realizar actividades de divulgación oral frente a actividades de divulgación escrita, lo cual lleva a pensar que quizás en el imaginario académico las actividades orales impliquen menor complejidad o dificultad, o que el personal académico se siente más cómodo o seguro con ellas.

El personal investigador encuestado no posee capacitación en el área de comunicación de la ciencia. En este sentido, se señala la necesidad de desarrollar procesos de formación que les ofrezcan las habilidades y capacidades necesarias para poder realizar adecuadamente actividades de comunicación científica en diversos contextos.

Los factores que facilitaron en el personal investigador encuestado la intención de participar en actividades de divulgación se encuentran asociados a la actitud, la norma subjetiva y el control percibido. No obstante, el sendero que más sobresale es el de control conductual percibido, por lo cual podría afirmarse que aquellos factores asociados a la autoeficacia, juegan un papel de gran relevancia al momento de involucrarse en actividades de divulgación (por ejemplo, la habilidad de responder preguntas de forma simple o la capacidad de exponer verbalmente las investigaciones a públicos con pocos conocimientos científicos).

Los factores principales que dificultan la divulgación de la ciencia son la falta de tiempo y la falta de reconocimiento por parte de régimen académico. Obstáculos que remiten a la necesidad de cuestionar la forma en la cual los procesos de divulgación son valorados y/o reconocidos en los sistemas de meritazgo universitarios.

Por último, desde el posicionamiento epistemológico de la presente investigación, es perentorio aportar a las comunidades académicas nuevos marcos referenciales que brinden una visión más amplia de los procesos de comunicación científica, desde la cual puedan cuestionar sus posicionamientos respecto a la valoración que realizan en torno a la divulgación de la ciencia.

\section{Referencias}

Agnella, Selena., De Bortoli, Andrea., Scamuzzi, Sergio., L'Astorina, Alba., Cerbara, Loredana., Valente, Adriana., \& Avveduto, Sveva. (abril, 2012). How and why scientists communicate with society: the case of physics in Italy. Trabajo presentado en la 12th International Public Communication of Science and Technology Conference. Florencia, Italia.

Ajzen, Icek. (2001). Nature and operation of attitudes. Annual Review of Psychology, 52, 27-58.

Ajzen, Icek. (2002a). Attitudes. En R. Fernández Ballesteros (Ed.). Encyclopedia of psychological assessment (pp.110-115). London: Sage Publications.

Ajzen, Icek. (2002b). Residual effects of past on later behavior: Habituation and reasoned action perspectives. Personality and Social Psychology Review, 6, 107-122. 
Ajzen, Icek. (2011). The theory of planned behavior: Reactions and reflections. Psychology \& Health, 26(9), 1113-1127.

Ajzen, Icek. (2012). The theory of planned behavior. En P. A. M. Lange, A. W. Kruglanski \& E. T. Higgins (Eds.), Handbook of theories of social psychology (pp.438-459). London: Sage.

Ajzen, Icek., \& Fishbein, Martin. (1975). Attitude-behavior relations: A theoretical analysis and review of empirical research. Psychological Bulletin, 84, 888-918.

Ajzen, Icek., \& Fishbein, Martin. (2008). Scaling and testing multiplicative combinations in the expectancy-value model of attitudes. Journal of Applied Social Psychology, 38, 2222-2247.

Ajzen, Icek., \& Gilbert Cote, Nicole. (2008). Attitudes and the prediction of behavior. In W. D. Crano., \& R. Prislin (Eds.), Attitudes and attitude change (pp. 289-311). New York: Psychology Press.

Ajzen, Icek., Joyce, Nicholas., Sheikh, Sana., \& Gilbert Cote, Nicole. (2011). Knowledge and the prediction of behavior: The role of information accuracy in the theory of planned behavior. Basic and Applied Social Psychology, 33(2), 101-117.

Andrews, Elisabeth., Weaver, Alex., Hanley, Daniel., Hovermill, Jeffrey., \& Melton, Ginger. (2005). Scientists and public outreach: participation, motivations, and impediments. Journal of Geoscience Education, 53(3), 281-293.

Anguera, María., Arnau, Jaime., Ato, Manuel., Martínez, Rosario., Pascual, Juan., \& Vallejo, Guillermo. (1998). Métodos de investigación en psicología. Madrid: Editorial Síntesis, S.A.

Barrantes, Rodrigo. (2007). Investigación. Un camino al conocimiento. San José, Costa Rica: Editorial EUNED.

Bentley, Peter., \& Kyvik, Svein. (2011). Academic staff and public communication: a survey of popular science publishing across 13 countries. Public Understand of Science, 20(1), 48-63.

Bradley, Keith. (2003). Intellectual capital and the new wealth of nations II. Business Strategy Review, $8(4), 33-44$.

Corrado, Michele., Pooni, Kam., \& Hartfree, Yvette. (2000). The Role of Scientists in Public Debate: FullReport. Londres: Market and Opinion Research International.

Duarte, Sergio. (2008). Factores determinantes de la actitud emprendedora investigativa en científicos del Paraguay. Revista Iberoamericana de Ciencia, Tecnología y Sociedad, 8(23), 67-87.

Edvinson, Leif., \& Malone, Michael. (2000). El capital Intelectual: cómo identificar y calcular el valor de los recursos intangibles de su empresa. Barcelona: Gestión.

Gantenberg, Julia. (abril, 2012). Between Ivory Tower and Spotlight. A Case Study on the Status Quo of PCST in Publicly Funded Research Centres. Trabajo publicado en la 12th International Public Communication of Science and Technology Conference. Florencia, Italia.

Kaliyaperumal, Karunamoorthi. (2004). Guideline for Conducting a Knowledge, Attitude and Practice (KAP) Study. AECS Illumination, 4(1), 7-9. Recuperado de http://laico.org/v2020resource/files/ guideline_kap_Jan_mar04.pdf

Kerliger, Fred., \& Lee, Howard. (2002). Investigación del comportamiento. México D.F.: McGraw-Hill.

Little, T.D., Cunningham, W.A., Shahar, G. \& Widaman, K.F. (2002). To Parcel or Not to Parcel: Exploring the Question, Weighing the Merits. Structural Equation Modeling, 9 (2), 151-173.

Little, T.D., Rhemtulla, M.,Gibson, K. \& Schoemann, A.M.(2013). Why the Items versus Parcels Controversy Need not be one. Psychological Methods, 18 (3), 285-300. doi: 10.1037/a0033266

Loaiza, Claudia. (abril, 2012). European scientists' public communication attitudes: scientists' views and experiences and the institutional, local and national influences determining their public engagement activities. Trabajo presentado en la 12th International Public Communication of Science and Technology Conference. Florencia, Italia.

Martín, María., \& Rey, Jesús. (2007). Papel de los científicos en la comunicación de la ciencia y la tecnología a la sociedad: actitudes, aptitudes e implicación. Madrid: Comunidad de Madrid y CSIC. 
Martínez, Manuel. (2008). La responsabilidad del investigador en la divulgación de la ciencia. Revista de Divulgación Científica y Tecnológica de la Universidad Veracruzana, 21(1). Recuperado de http://www.uv.mx/cienciahombre/revistae/vol21num1/articulos/responsabilidad/index.html

Méndez, Pablo., Valdez, Rosario., Velázquez, Leonardo., Rivera, Leonor., \& Salmerón, Jorge. (2003). Violencia contra la mujer: conocimiento y actitud del personal médico del Instituto Mexicano de Seguro Social, Morelos, México. Revista Salud Pública de México, 45(6), 472-482.

Mújica, Alejandro., Guido, Priscila., \& Mercado, Serafín. (2011). Actitudes y comportamiento lector: una aplicación de la teoría de la conducta planeada en estudiantes de nivel medio superior. Liberabit, 17(1), 77-84.

Osgood, Charles., Suci, George., \& Tannnenbaum, Percy. (1986). Capítulo 13: Medición de actitudes. En G. Summers (Comp.). Medición de actitudes. México D.F.: Editorial Trillas.

Peters, Hans., Brossard, Dominique., Cheveigné, Suzanne., Dunwoody, Sharon., Kallfass, Monika., Miller, Steve., \& Tsuchida, Shoji. (2008). Science Communication. Interactions with the Mass Media. Sciencemag, 321, 204-205.

Reyes, Luis. (2007). La teoría de la acción razonada: implicaciones para el estudio de las actitudes. Investigación Educativa, 7, 66-77.

Rivera, José. (2002). Ciencia y divulgación. Revista Biomédica, 13(2), 152-153.

Torres-Albero, Cristóbal., Fernández-Esquinas, Manuel., Rey-Rocha, Jesús., \& Martín-Sempere, María José. (2011). Dissemination practices in the Spanish research system: scientists trapped in a golden cage. Public Understand of Science, 20(1), 12-25. 
